\title{
Temperature dependence of the zero point kinetic energy in ice and water above room temperature
}

\author{
R. Senesi ${ }^{\mathrm{a}, \mathrm{b}, *}$, G. Romanelli ${ }^{\mathrm{a}}$, M.A. Adams ${ }^{\mathrm{c}}$, C. Andreani $^{\mathrm{a}}$ \\ a Università degli Studi di Roma "Tor Vergata", Dipartimento di Fisica and Centro NAST, via della Ricerca Scientifica 1, 00133 Roma, Italy \\ ${ }^{\mathrm{b}}$ CNR-IPCF Sez. di, Messina, Italy \\ ' ISIS Facility, Science and Technology Facilities Council, Harwell Science and Innovation Campus Chilton, Didcot OX11 OQX, UK
}

\section{A R T I C L E I N F O}

\section{Article history:}

Available online 9 October 2013

\section{Keywords:}

Nuclear quantum effects

Zero point kinetic energy

Hydrogen bond

Proton potential

Inelastic neutron scattering

\begin{abstract}
A B S T R A C T
By means of Deep Inelastic Neutron Scattering we determined the temperature dependence of the proton kinetic energy in polycrystalline ice Ih between $5 \mathrm{~K}$ and $271 \mathrm{~K}$. We compare our results with predictions form Path Integral quantum simulations and semiclassical quasi-harmonic models with phasedependent frequencies. The latter show the best agreement with the experiment if the librational contribution is properly taken into account. The kinetic energy increase with temperature in ice is also found to be approximately a factor $\sim 5$ smaller than in the case of liquid water above room temperature, highlighting the role played by anharmonic quantum fluctuations in the two phases.
\end{abstract}

(c) 2013 Elsevier B.V. All rights reserved.

\section{Introduction}

Measurements of momentum distributions are a matter of widespread interest because of their connection to the theories of Bose/Fermi liquids, anharmonic solids [1], and in the case of water, to provide valuable experimental evidence relating to the hydrogen-bond potential energy surfaces [2-4]. The most direct method to measure the momentum distribution is Deep Inelastic Neutron Scattering [5,6], with experimental methods mainly developed at the ISIS pulsed neutron source and following a theoretical study by Gunn et al. [7-10]. This technique allows the exploration of neutron scattering as a probe of single-particle excitations in condensed matter. In this work we consider the second moment of the momentum distribution, the mean kinetic energy $\left\langle E_{K}\right\rangle$. In contrast to the potential energy, the kinetic energy of a condensed system has distinct properties in the classical regime (equipartition), in the liquid phases, in the crystal phase (Debye or Einstein-like description). Previous to DINS, the single particle mean kinetic energy has been inaccessible to direct measurements; indeed indirect inferences has been available from Debye-Waller factors and phonon spectra [11], or from the mass derivative of the Helmoltz free energy [12]. In the case of molecular hydrogen in the condensed phase, inelastic neutron scattering was used to probe the decoupling approximation between the

\footnotetext{
* Corresponding author at: Università degli Studi di Roma "Tor Vergata", Dipartimento di Fisica and Centro NAST, via della Ricerca Scientifica 1, 00133 Roma, Italy. Tel.: +39 06 72594549; fax: +39062023507.

E-mail address: roberto.senesi@uniroma2.it (R. Senesi).
}

molecular center of mass and internal vibrations to determine the molecular kinetic energy [13-15].

Studies of the temperature dependence of $\left\langle E_{K}\right\rangle$ have been devoted to the understanding of the changes that occur in the dynamical properties as the system goes from the classical to the quantum regime. Indeed for fluid helium at low, intermediate, and high densities with respect to the $\lambda$ point, the temperature dependence reveals a rich behaviour on the details of the interatomic interactions and deviations from harmonicity [16-19]. DINS measurements on He at intermediate densities, along an isochore as a function of temperature, showed that harmonic motion in the cage formed by the near neighbours could account for the zero-point kinetic energy of the atoms and that a single frequency Einstein-oscillator model could adequately describe the temperature behaviour of the kinetic energy [17]. For higher densities, $e$. $g$. twice that at the $\lambda$ point, this picture is revised, taking into account a temperature-density dependent oscillator frequency [18]. At the extreme of low densities, about half of that at the $\lambda$ point, the excess of kinetic energy with respect to the classical expectation, falls off very slowly with increasing temperature [16]. The slow decay with temperature reflects the contribution of binary collisions from steep interatomic potentials, i.e. the relevance of the hard-core component in the interaction potential in helium [19]. A special application of the above method was devoted to the case of superfluid helium, where the temperature dependence of $\left\langle E_{K}(T)\right\rangle$ has been determined to infer the zero-momentum condensate fraction, in agreement with previous determinations and with theoretical predictions [20]. In the case of neon, where anharmonicities are still expected to be relevant, a series of DINS experiments [21] to measure $\left\langle E_{K}(T)\right\rangle$, assessed the most direct 
and model-free determination of anharmonicities in this system. Fradkin et al. [11] measured the kinetic energy of condensed $\mathrm{Ar}$ at low temperatures, in a regime below equipartition; they pointed out how a purely harmonic description, in which $\left\langle E_{K}\right\rangle$ is proportional to $\left\langle u^{2}\right\rangle$, the mean-squared displacement, cannot reproduce the $\left\langle E_{K}(T)\right\rangle$ behaviour; a self -consistent-average-phonon model, with temperature-dependent average phonon frequencies, or more sophisticated descriptions such as Path Integral simulations, were needed to provide a quantitative agreement with experiments [11]. Similarly to the case of the noble-gas helium, condensed lithium shows a slow decay of the excess kinetic energy with temperature [22], consistently with Path Integral quantum simulations [23]. In particular, Evans et al. [22] reported a kinetic energy approximately $10 \%$ higher than that predicted by a harmonic model incorporating a density of states from previous phonon measurements. A quartic anharmonic perturbation to a harmonic potential was employed to model satisfactorily the observed $\left\langle E_{K}\right\rangle$ of lithium from DINS measurements [22].

In the case of hydrogen-containing heteronuclear polyatomic molecular systems, the centre of mass kinetic energy should closely follow the classical equipartition, although experimental assessments, with special regards to isotopic effects, are still missing. The most prominent character of quantum fluctuations in these systems is instead shown by kinetic energy of the proton, the lightest atomic species in the molecule. Indeed $\left\langle E_{K}\right\rangle$ of the proton arises from the interplay between the "internal" vibrations, such as stretching and bending, and "external" vibrations, such as roto-translational motions [24,25]. Due to the different energies associated to internal and external motions, the internal contribution to $\left\langle E_{K}(T)\right\rangle$ is predominant over the roto-translational one. It is expected that in the condensed phases the internal contribution is only weakly affected by intermolecular interactions and temperature, while the much smaller proton kinetic energy contributions coming from the molecular external modes are influenced by intermolecular interactions [25].

On this basis it appears that an uncorrelated, decoupled, description of the $\left\langle E_{K}\right\rangle$, in terms of the sum of internal and external contributions, should be able to capture all the relevant underlying physics to model the temperature behaviour of $\left\langle E_{K}(T)\right\rangle$. Here the internal vibrations are meant just as local modes, pertaining to one single molecule only, while the complexity of the many-body problem is retained by the external term, acting as an additional contribution to the more relevant internal components. This framework has been applied, for example, to fluid and solid $\mathrm{H}_{2} \mathrm{~S}$, providing a good agreement between the DINS determinations of $\left\langle E_{K}(T)\right\rangle$ and the values calculated using the decoupling approximation described above [25].

Water, with its extended network of hydrogen bonds, and the destructuring effects from quantum fluctuations associated to the water's protons, is the most relevant test-bed H-bonded system for assessing the kinetic energy descriptions outlined above, and to probe the peculiar deviations associated to anharmonicities in the proton's motions [26]. The extent to which quantum fluctuations affect water's structure and dynamics remains a subject of considerable debate [26]; theoretical predictions suggest that the zero point fluctuations in the anharmonic $\mathrm{O}-\mathrm{H}$ stretching coordinate increase the average $\mathrm{O}-\mathrm{H}$ bond length and give the water molecule a larger average dipole moment than it would otherwise possess [27]. It is thus expected that, starting from the model of an isolated molecule, such as what can be encountered in the vapour phase, intermolecular interactions induce changes in the internal and external vibration frequencies, and that the proton kinetic energy is the result of competing effects with various degrees of compensation depending on the temperature and on the phase [26-29,24,2]. For example, in the supercritical phase, DINS measurements $[29,4,30]$ showed that as the density increases in lowering the temperature from supercritical to ambient conditions, the intra-molecular stretching modes become softer, while the external modes - mainly hindered rotations and librations- become harder. These two changes seem to compensate each other almost in an exact way, providing kinetic energy values similar to those calculated for protons in an isolated molecule $[30,4,29]$. On the other hand, recent sophisticated electrostatic models for water cannot provide an even qualitatively accurate description of the temperature dependence of the $\mathrm{O}-\mathrm{H}$ stretching component to the proton's $\left\langle E_{K}(T)\right\rangle$ [2]. The relative lowering in kinetic energy at temperatures below approximately $500 \mathrm{~K}$ reflects the softening of the vibrational stretching potential due to $\mathrm{H}$-bonding, and intermolecular interactions in general, which are not reproduced by up-to-date electrostatic models. Burnham et al., in Ref. [2] express their doubts whether "any of the existing models are accurate enough to even qualitatively account for the environmentally dependent kinetic energy changes in the vibrational modes". We stress that, although the first DINS experiment at $\mathrm{eV}$ energies was carried out on liquid water in 1968 [31], all the subsequent experimental and theoretical work on $\left\langle E_{K}(T)\right\rangle$ in the condensed phases of water is still focussed towards a comprehensive description of nuclear quantum effects in this prototype H-bonded system, see for example Refs. (and references therein $[3,32-34,2,35,4,5]$ ).

In the solid phase, a recent study assessed the anomalous isotope effects on the thermal expansion of ice [36], which were shown to originate on competing anharmonicities in the vibrational modes of the system. These isotope effects are most evident in their temperature dependence, in that the volume difference between heavy and light ice increases with temperature [36]. Moreh and Nemirovski [24] calculated $\left\langle E_{K}(T)\right\rangle$ of the proton in ice Ih between $5 \mathrm{~K}$ and $269 \mathrm{~K}$, using optical vibration frequencies from the literature, assuming the harmonic approximation and decoupling between the degrees of freedom of translation, rotation-libration, and internal vibrations. They obtained good agreement with DINS data at $5 \mathrm{~K}$ [37] but not at $269 \mathrm{~K}$ [38], assuming free rotation of the entire molecule. More recently, Finkelstein and Moreh [39] reported new calculations on ice at ambient (and high) pressure, which account for a revised evaluation of the librational component from the literature, obtaining a $\sim 10 \mathrm{meV}$ increase in the calculated kinetic energy. This is not surprising, given the importance of the coupling and modulation of the stretching by librational motions, with various degrees of anharmonicity [40]. Ramirez and Herrero determined $\left\langle E_{K}(T)\right\rangle$ using quantum Path Integral simulations based on ab initio Density Functional calculations [41-43], obtaining values somewhat larger than the free-rotations harmonic calculations by Moreh and Nemirovski [24]. Path integral simulations were also used to determine $\left\langle E_{K}(T)\right\rangle$ in amorphous ice [44], with a $\sim 2 \mathrm{meV}$ decrease in $\left\langle E_{K}(T)\right\rangle$ with respect to polycrystalline ice Ih, as a consequence of the softer potentials and larger quantum delocalization of hydrogen in amorphous ice, as compared with ice Ih at the same temperature [44].

In this work we present DINS measurements of the proton's $\left\langle E_{K}(T)\right\rangle$ on polycrystalline ice $\mathrm{Ih}$, in the temperature range $5 \mathrm{~K}$ $\leqslant \mathrm{T} \leqslant 70 \mathrm{~K}$. These are used together with previously measured $\left\langle E_{K}\right\rangle$ at $\mathrm{T}=271 \mathrm{~K}$ for ice [45], to derive the temperature behaviour of $\left\langle E_{K}(T)\right\rangle$. The results are compared with the theoretical predictions outlined above and with previous measurements in the liquid above room temperature. The different temperature dependence of the kinetic energy in the solid and liquid phases is derived and discussed.

Section II reports the experimental details and in Section III we discuss and summarize the main similarities and discrepancies between our results and those given in some earlier works and the theoretical predictions. 


\section{Experiment}

The DINS measurements have been carried out on the VESUVIO time of flight inverse geometry spectrometer [8] at the ISIS spallation neutron source (Rutherford Appleton Laboratory, UK). VESUVIO operates in the Impulse Approximation [5] regime where the incident neutron wavelengths are much less than the inter-atomic spacing and thus atoms scatter incoherently, with scattered intensity being the sum of intensities from individual atoms in the sample, with conservation of the total kinetic energy and momentum of the neutron and the atom. In the IA regime the inelastic neutron scattering cross section is related in a simple way to the atomic momentum distribution, $n(\mathbf{p})$. The neutron scattering function $S_{\mathrm{IA}}(\mathbf{q}, \omega)$ is

$\frac{h q}{M} S_{\mathrm{IA}}(\mathbf{q}, \omega)=J_{\mathrm{IA}}(y, \hat{\mathbf{q}})=\int n(\mathbf{p}) \delta(y-\mathbf{p} \cdot \hat{\mathbf{q}}) \mathrm{d} \mathbf{p}$

where $(\mathbf{q}, \omega)$ are the wave vector and energy transfers, $M$ is the mass of the struck atom, $y=\frac{M}{h q}\left[\omega-\frac{h q^{2}}{2 M}\right]$ is the particle momentum along the $\hat{\mathbf{q}}$ direction, and $J_{\mathrm{IA}}(y, \hat{\mathbf{q}})$ is the neutron Compton Profile (NCP) [5].

When the sample is isotropic, the particle momentum distribution only depends on the modulus of $\mathbf{p}$, and the $\hat{\mathbf{q}}$ direction is immaterial, so the NCP is simply $J_{\mathrm{IA}}(y)=2 \pi \int_{|y|}^{\infty} p n(p) \mathrm{d} p$. The kinetic energy is directly proportional to the second moment of the NCP $\left\langle E_{K}\right\rangle=\frac{3 h^{2}}{2 M} \int_{-\infty}^{\infty} y^{2} J_{I A}(y) d y=\frac{3 h^{2}}{2 M} \sigma^{2}$.

The NCP is broadened by finite- $q$ correction terms $\Delta J(y, q)$, and by convolution with the instrumental resolution function $R(y, q)$, so the experimental NCP, $F(y, q)$, is [5]:

$F(y, q)=\left[J_{\mathrm{IA}}(y)+\Delta J(y, q)\right] \otimes R(y, q)$.

The instrument is equipped in the forward direction with Yttrium Aluminum Perovskite (YAP) scintillators located at a distance $L_{1}$, ranging between $0.5 \mathrm{~m}$ and $0.75 \mathrm{~m}$ from sample position, in the angular range $32.75^{\circ}$ to $72.5^{\circ}$. At each scattering angle the energy of the scattered neutrons, $E_{1}$, is selected by using Au analyzer foils $\left(E_{1}=4897 \mathrm{meV}\right)$. For forward scattering angles, such as those used for proton studies, the instrument operates using the Foil Cycling (FC) technique [46], accessing the kinematical range: $25 \AA^{-1} \leqslant q \leqslant 600 \AA^{-1}$, and $2.5 \mathrm{eV} \leqslant h \omega_{r} \leqslant 30 \mathrm{eV}$, where $h \omega_{r}=h^{2} q_{r}^{2} / 2 M$, where $q_{r}$ is the wave vector transfer at the recoil peak.

The sample was held in an Al container with internal walls coated with polytetrafluoroethylene (PTFE), with an internal sample volume $6.3 \times 6.3 \times 0.1 \mathrm{~cm}^{3}$, equipped with $\mathrm{Rh} / \mathrm{Fe}$ sensors for temperature monitoring. The DINS measurements were carried out for temperatures between $5 \mathrm{~K}$ and $71 \mathrm{~K}$. For each temperature the data acquisition had a duration of approximately $36 \mathrm{~h}$, corresponding to $\sim 6500 \mu \mathrm{A}$ hours of integrated proton current.

Data reduction on the raw time of flight data, and the determination of the instrumental resolution functions, have been carried out using procedures described in details Ref. [45, and references therein]. As an example of the quality of data for each of the 48 detectors used in the experiment, we report in Fig. 1 the raw time of flight data recorded by a single detector, after gamma background correction [47], together with the evaluation of the multiple scattering contribution, for ice Ih at $\mathrm{T}=5 \mathrm{~K}$.

Data have been then transformed $y$ - space using standard routines and then fitted by the minimization of:

\footnotetext{
${ }^{1}$ For consistency with previous literature and ease of notation we write the momentum as a wave vector.
}

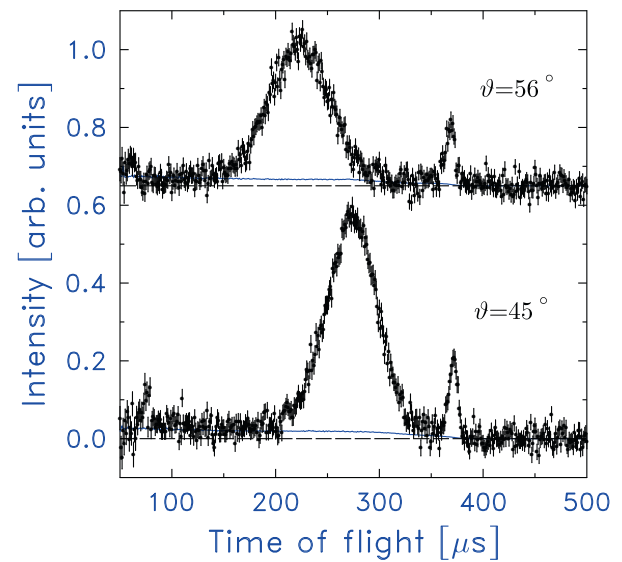

Fig. 1. Time of flight DINS spectra for ice Ih at $\mathrm{T}=5 \mathrm{~K}$, for detectors at scattering angles $\vartheta=45^{\circ}$ and $\vartheta=56^{\circ}$ (the latter is shifted upwards for clarity), dots with error bars, after gamma background correction [47]. Monte Carlo evaluation of multiple scattering contributions are reported as blue continuous lines [[45] and references therein]. The most intense peak is due to the proton signal, while the less intense peak is due to the contributions from oxygen (ice) and aluminium (sample container). (For interpretation of the references to colour in this figure caption, the reader is referred to the web version of this article.)

$\chi^{2}=\sum_{l} \sum_{i} \frac{\left(F_{l}^{t h}\left(y_{i}, q_{i}\right)-F_{l}\left(y_{i}, q_{i}\right)\right)^{2}}{\epsilon_{l, i}^{2}}$

where the model function is $F_{l}^{t h}\left(y_{i}, q_{i}\right)=\left[J\left(y_{i}\right)+\Delta J_{l}\left(y_{i}, q_{i}\right)\right] \otimes R_{l}\left(y_{i}, q_{i}\right)$. Here $J\left(y_{i}\right)$ is described by a Gauss-Hermite line shape [45, and references therein]:

$J(y)=\frac{e^{\frac{-y^{2}}{2 \sigma^{2}}}}{\sqrt{2 \pi} \sigma}\left[1+\sum_{n=2}^{\infty} \frac{a_{n}}{2^{2 n} n !} H_{2 n}\left(\frac{y}{\sqrt{2} \sigma}\right)\right]$.

where the fitting parameters are $\sigma$, the standard deviation of $J(y)$, and $a_{n}$, the coefficients of the Hermite polynomials, $H_{2 n}$. The deviations from the Impulse Approximation are accounted for by the following expression:

$\Delta J_{l}\left(y_{i}, q_{i}\right)=\frac{e^{\frac{-y_{i}^{2}}{2 \sigma^{2}}}}{\sqrt{2 \pi} \sigma} \frac{c 1}{q_{i}} H_{3}\left(\frac{y_{i}}{\sqrt{2} \sigma}\right)$

where $c 1$ is a fitting parameter [45, and references therein].

In the $\chi^{2}$ expression of Eq. $3, l$ represents the detector index, the index $i$ represents the $y$ value at the $i$ th bin, and $\epsilon_{l, i}^{2}$ is the error for each data point. The double sum over $l$ and over $i$ reflects the relevant property:

$J(y)$ is unique for all detectors; $\Delta J_{l}(y, q)$ varies across detectors due to the different $q$ values accessed [48], but $c 1$ is independent of $q$ and is unique for all detectors; $R_{l}\left(y_{i}, q_{i}\right)$ varies across detectors, but is a known function from the Monte Carlo evaluation [45][and references therein].

\section{Results and discussion}

Fig. 2 reports the detector-averaged Neutron Compton profile, $\bar{F}(y)=\Sigma_{l=1}^{n} F_{l}(y, q) \frac{1}{n}$, where $n=48$ is the total number of detectors used in the experiment, for ice Ih at $\mathrm{T}=5 \mathrm{~K}$. This represents an example of the data quality of such measurements, which take advantage of the narrow resolution for proton momentum available on VESUVIO.

Here the ratio between the FWHM of $\bar{R}(y)$ and $\bar{F}(y)$ is approximately $15 \%$, i.e. a working condition similar to that of X-ray Compton scattering [49]. The value for the kinetic energy derived from the fit is $\left\langle E_{K}(T=5 K)\right\rangle=(150.87 \pm 1.50) \mathrm{meV}$, higher than the calculation of $144 \mathrm{meV}$ and a previous experimental determination [24,37]: 


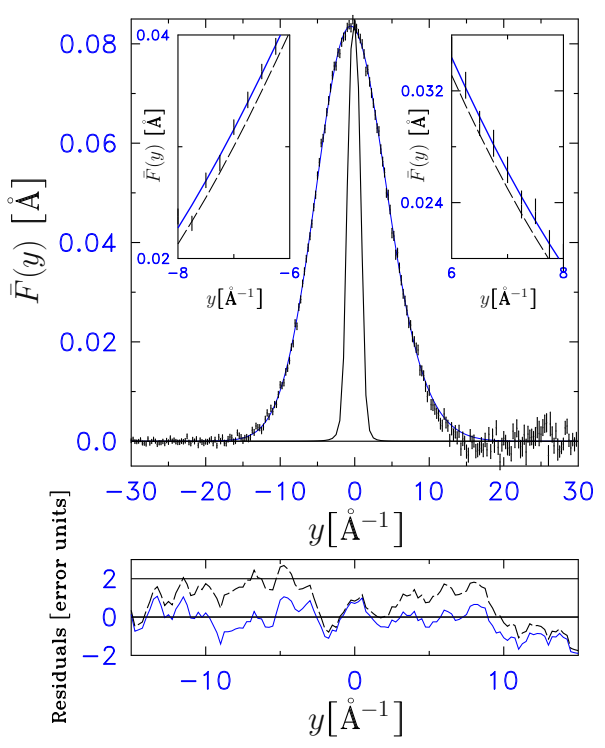

Fig. 2. Main panel: detector-averaged $\bar{F}(y)$ for ice $\mathrm{Ih}$ at $\mathrm{T}=5 \mathrm{~K}$ (circles with error bars), together with the (detector-averaged) best fit (blue continuous line, corresponding to $\left\langle E_{K}\right\rangle=150.87 \mathrm{meV}$ ). The detector-averaged resolution, $\bar{R}(y)$ is reported as a black continuous line. The best fit corresponding to a kinetic energy $\left\langle E_{K}\right\rangle=144 \mathrm{meV}$, following the calculations of Moreh et al. [24] is reported as a black dashed line. The two insets in the main panel reports the data and the two best fits corresponding to $\left\langle E_{K}\right\rangle=150.87 \mathrm{meV}$ and $\left\langle E_{K}\right\rangle=144 \mathrm{meV}$, in the intermediate momentum ranges, highlighting the goodness of the two fits. The lower panel reports the differences between data and fit, divided by the error bar, in the momentum range $-15 \AA^{-1} \leqslant y \leqslant 15 \AA^{-1}$. (For interpretation of the references to colour in this figure caption, the reader is referred to the web version of this article.)

indeed a fit carried out imposing $\left\langle E_{K}\right\rangle=144 \mathrm{meV}$ shows larger residuals at the low and intermediate momentum values, with respect to a fitting line shape with the value of $\left\langle E_{K}\right\rangle=150.87 \mathrm{meV}$, as shown in Fig. 2. An example of the temperature dependence of the NCP is reported in Fig. 3 . This shows the $\bar{F}(y)$ for ice Ih at $5 \mathrm{~K}$ and a previous measurement at $271 \mathrm{~K}$ [45] under similar experimental configurations. The temperature increase induces a broadening in $\bar{F}(y)$, which is then reflected in an increase of $\left\langle E_{K}(T)\right\rangle$.

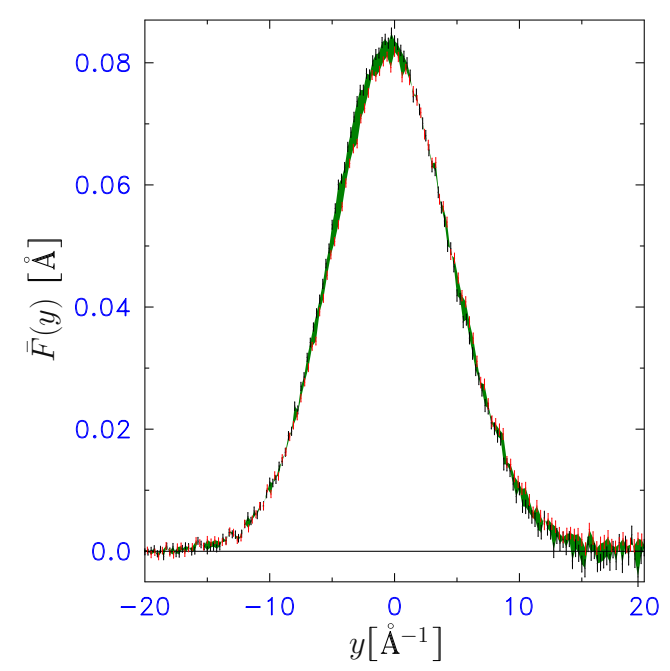

Fig. 3. Main panel: detector-averaged $\bar{F}(y)$ for ice Ih at $\mathrm{T}=5 \mathrm{~K}$ (black dots with error bars), and ice Ih at $\mathrm{T}=271 \mathrm{~K}$ from a previous measurement carried out under the same experimental configuration [45] (red dots with error bars). The difference between the two profiles is highlighted by the green colour filling. (For interpretation of the references to colour in this figure caption, the reader is referred to the web version of this article.)
As shown in the Introduction Section, there are two main approaches for the theoretical evaluation of $\left\langle E_{K}(T)\right\rangle$. Recent computational methods make use of advanced methodologies for the inclusion of nuclear quantum effects, such as Path Integral Car-Parrinello Molecular Dynamics (PICPMD) [32], or Path Integral Molecular Dynamics employing empirical potential models for water [43], or coupled with Generalized Lanvegin equations [3]. The other method consists in using empirical models assuming that $\left\langle E_{K}(T)\right\rangle$ is obtained from a set of decoupled quantum harmonic oscillators whose frequency is derived from optical data and/or measured vibrational density of states [39,29,24]. Typically, translational degrees of freedom are assumed classical, while a quantum harmonic description is used for librational, bending and stretching vibrations $[39,29,24]$ :

$$
\begin{aligned}
\left\langle E_{K}(T)\right\rangle= & S_{t} \frac{3}{2} k_{B} T+S_{l i b} \frac{h \omega_{l i b}}{4} \operatorname{coth}\left(\frac{h \omega_{\text {lib }}}{2 k_{B} T}\right)+S_{\text {ben }} \frac{h \omega_{\text {ben }}}{4} \\
& \times \operatorname{coth}\left(\frac{h \omega_{\text {ben }}}{2 k_{B} T}\right)+S_{\text {str }} \frac{h \omega_{\text {str }}}{4} \operatorname{coth}\left(\frac{h \omega_{\text {str }}}{2 k_{B} T}\right)
\end{aligned}
$$

where $S_{\alpha}$ are the kinetic energy fractions shared by the proton in translation, libration, bending, stretching, respectively, and $h \omega_{\alpha}$ are the corresponding energies derived from complementary spectroscopic data, for each phase/thermodynamic state investigated. It is immediately apparent that the use of phase/temperature dependent energies, $h \omega_{\alpha}$, reflects the deviations from purely harmonic description, which however is inherent in Eq. (6) above. Eq. (6) provides also the harmonic-model temperature dependence of the kinetic energy, which is determined by the combination of the $\propto \operatorname{coth}\left(\frac{h \omega_{\alpha}}{2 k_{B} T}\right)$ dependent contributions, bearing in mind that this model neglects the interactions between translational, librational and vibrational degrees of freedom, the presence of different environments in the liquid, and the inhomogeneous broadening that follows [40].

In their phonon calculations of ice Ih at $269 \mathrm{~K}$, Lin et al. [50] determined the vibrational dynamics and a quasiharmonic multivariate Gaussian momentum distribution. They found a difference of approximately $14 \mathrm{meV}$ between the kinetic energy value from the end-to-end distribution from PICMD (143 meV) and that obtained from a lattice dynamics $(157.5 \mathrm{meV})$. They concluded that this difference arises from the anharmonicity due to H-bonding, mostly in the stretching and librational vibrations; the anharmonicity of the ground state was determined by a calculation of the potential energy surface along the $\mathrm{H}$-bond direction, showing a cubic dependence on proton displacement.

Differences in the calculated values of $\left\langle E_{K}(T)\right\rangle$ using Path Integral simulations are in general ascribed to the relative uncertainties associated the choice of the empirical potential models for water $[43,3]$ or to the density functional theory approximations in ab initio calculations [45]. The empirical decoupled harmonic models of Refs. [39,29,24], can instead be regarded as "ex post" quasiharmonic descriptions, since deviations from harmonicity are implicitly assumed in the phase/state -dependent principal frequencies and/or density of states from spectroscopic measurements.

From the experimental point of view, DINS measures almost directly the kinetic energy of the equilibrium state, that is the kinetic energy of ground and excited states for the low energy vibrations (comparable with $k_{B} \mathrm{~T}$ ) and only the ground state for the intramolecular bending and stretching modes of water molecule (because at the typical investigated temperatures these higher energy modes are not excited at all). On the other hand spectroscopic measurements, for example Inelastic Neutron Scattering (INS) in energy loss configuration, measure the transition from the ground state to the first excited state, and in case the system is 
anharmonic, a calculation of $\left\langle E_{K}(T)\right\rangle$ based on spectroscopic data may be in general different compared to the result of $\left\langle E_{K}(T)\right\rangle$ from DINS even at low temperatures.

The $\left\langle E_{K}(T)\right\rangle$ values from the present work, together with those reported in Refs. [39,24,42] are shown in Fig. 4). The present measurements show that the proton kinetic energy in ice is essentially determined by its zero point value (approximately $151 \mathrm{meV}$ at $\mathrm{T}=5 \mathrm{~K}$ ), with a slight increase (about $0.02 \mathrm{meV} / \mathrm{K}$ between $5 \mathrm{~K}$ and $271 \mathrm{~K}$ ) with temperature. In the whole temperature range in the solid, $\left\langle E_{K}(T)\right\rangle$ is approximately five times larger than the classical values, highlighting the role played by nuclear quantum effects on the protons in ice. Thermal excitation acts as a relatively small perturbation to the quantum kinetic energy of the proton $[43,39]$.

As a general comment, we note that the Path Integral quantum simulations of Ref. [42], which include an anharmonic flexible potential for water, are in better agreement with the present data, with respect to the harmonic model of Ref. [24] assuming free rotations. Path Integral simulations provide in some cases slightly different results, for example in ice Ih near the triple point, ranging between $143 \mathrm{meV}$ [32,45] and $150 \mathrm{meV}$ [42], reflecting the uncertainties and approximations of these methods. We note that the explicit inclusion of anharmonicity in Ref. [42], together with the absence of the decoupling between degrees of freedom, improves the quantitative agreement with DINS data, as compared to the harmonic model. On the other hand, the new results by Finkelstein and Moreh [39], which account for the librational contributions are in the best agreement with the present data, highlighting the role played by intermolecular interactions and providing a probe of the sensitivity of the DINS technique to intermediate range order properties of the $\mathrm{H}$-bond network [50]. It is not possible here to identify the sources responsible for the underestimation of $\left\langle E_{K}(T)\right\rangle$ in the Path Integral simulations with respect to the experiment, while the shortcomings due to the harmonic/decoupling calculations appears to show probably compensating effects that result in better agreement with the present measurements.

In order to garner a more comprehensive picture of the kinetic energy in the condensed phase we report in Fig. 4) also the data for the liquid from previous measurements from room temperature to the supercritical phase $[4,51]$, where we recall that the liquid at room temperature has been measured also in Refs. [52,34,38], with

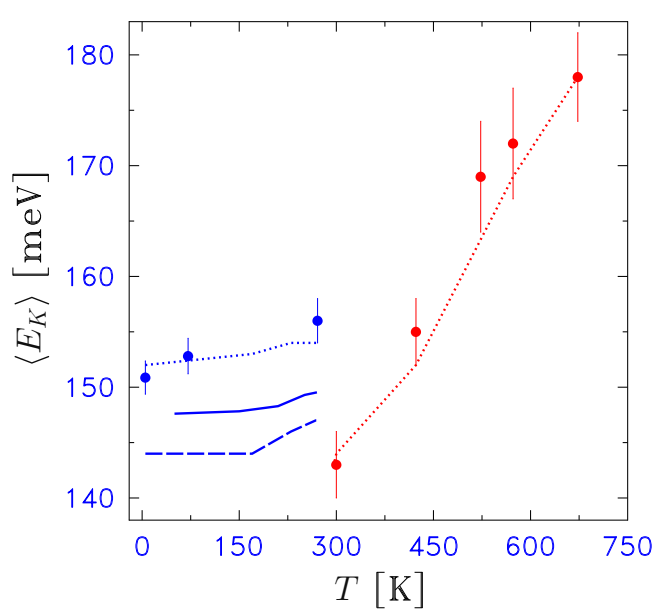

Fig. 4. (a): $\left\langle E_{K}(T)\right\rangle$, for ice Ih (blue full circles with error bars). Values from Moreh et al., [24], assuming free rotations (blue dashed line), from Finkelstein and Moreh [39] including librations (blue dotted line); Path Integral results from Herrero et al., [[42] and references therein] (blue continuous line). Data from previous measurements on stable liquid and supercritical water [[51] and references therein] are reported as red circles with error bars, together with the values from Moreh et al., [24](red dotted line). (For interpretation of the references to colour in this figure caption, the reader is referred to the web version of this article.) consistent results. These start from an initial value lower than ice at $\mathrm{T}=271 \mathrm{~K}$ and then show an increase with a stronger T-dependence (approximately $0.1 \mathrm{meV} / \mathrm{K}$ ) with respect to the solid. This temperature dependence is well reproduced by the data of Ref. [24]. However, as pointed out by Moreh et al., [24], the harmonic-decoupled model agrees with experiments for the liquid phase only if free rotations are assumed. If the librational contribution is accounted for, then the calculated kinetic energies would be shifted upwards by about $10 \mathrm{meV}$ (for example $\left\langle E_{K}(T=300 \mathrm{~K})\right\rangle=$ $154 \mathrm{meV})$, i. e. with a $\sim 7 \%$ overestimation throughout the temperature range. This is opposite to what is found for ice: the inclusion of librational contributions improves agreement for ice but worsens the agreement for the liquid. It is likely that the absence of coupling between intra- and intermolecular contribution is the main shortcoming of the above calculation to comprehensively reproduce both condensed phases. This confirms the picture of the dense, flexible, H-bond network model of the liquid as opposed to the more rigid solid, with variable degrees of anharmonic modulations of intramolecular stretching by librations [40].

In this context it should be noticed that the difference in $\left\langle E_{K}\right\rangle$ from ice at $271 \mathrm{~K}$ to the liquid at room temperature should be further investigated, both experimentally and theoretically. Indeed already in the Path Integral calculations in Reference [43] it was shown that, at constant temperature, the difference of $\left\langle E_{K}\right\rangle$ was always larger in ice Ih than in water by approximately $1 \mathrm{meV}$, while in the experiments this difference is larger, $\sim 13 \mathrm{meV}$. Recently, measurements of the hydrogen-projected density of states have allowed an independent determination of $\left\langle E_{K}\right\rangle$ [53], showing a $\sim 8 \mathrm{meV}$ difference between the solid and the liquid, in qualitative agreement with the present work. Increased kinetic energy in the solid with respect to the liquid is also reported in Path Integral simulations of water using rigid models [54]. Such combined efforts should help to shed some light on the effects of isotopic substitution (H-D) or the behaviour of $\left\langle E_{K}(T)\right\rangle$ in the region below room temperature or near the triple point, where the large discrepancies between experiments on water's protons and deuterons, and theories, are still unexplained [33,35]; we note, in passing, that indications of slight if not almost negligible differences in the deuteron kinetic energies were reported between room temperature liquid and solid heavy water close to the triple point [55], the solid showing in this case a slightly lower kinetic energy than the liquid. Values inferred from macroscopic thermodynamic free energy data on the proton kinetic energy in ice at $269 \mathrm{~K}$ and liquid water at $300 \mathrm{~K}$ predict $\mathrm{a} \sim 0.5 \mathrm{meV}$ increase from the solid to the liquid [56]. There is clearly a case to reconcile the results for the theory from Path integral simulations (higher kinetic energy, $\sim 1 \mathrm{meV}$, in the solid), the experiments (higher kinetic energy, $\sim 13 \mathrm{meV}$, in the solid), and the values from thermodynamic data (higher kinetic energy, $\sim 0.5 \mathrm{meV}$, in the liquid).

\section{Conclusions}

We have measured the proton mean kinetic energy in ice Ih at low temperatures and combined with previously measured data close to the triple point and in the liquid state, with the aim of determining and its temperature dependence, $\left\langle E_{K}(T)\right\rangle$ in a wide temperature range.The proton kinetic energy in ice is essentially determined by its zero point value, of the order of $150 \mathrm{meV}$, with a slight increase of about $0.02 \mathrm{meV} / \mathrm{K}$ between $5 \mathrm{~K}$ and $271 \mathrm{~K}$ with temperature. Three different predictions have been compared with the data, namely Path Integral simulation with anharmonic flexible water models $[42,43]$, and empirical models assuming harmonicity and decoupling between the degrees of freedom of proton motions, with and without explicit account of librational contributions to the proton's kinetic energy [24,39]. The best 
agreement with the experiment is shown by the harmonicdecoupled model of Ref. [39] with the inclusion of librational contributions, whereas the Path Integral results slightly underestimate the experimental values [42,43], but are in better agreement with respect to the harmonic-decoupled calculation that neglects librations and assumes free rotations [24]. The situation is reversed for the liquid, where the empirical harmonic-decoupled model neglecting librations improves the agreement while the inclusion of librations overestimates the kinetic energies of $\sim 7 \%$ with respect to the experiments $[24,39]$. As far as the temperature dependence is concerned, the kinetic energy increase with temperature in ice is found to be approximately a factor $\sim 5$ smaller than in the case of liquid water above room temperature. This is consistent with a picture where the kinetic energy is dominated by the ground state contributions, with the thermal contributions affecting mostly the high-temperature liquid upon approaching the supercritical phase. Differences in the ground state contributions between the solid and the liquid are evident in $\mathrm{a} \sim 13 \mathrm{meV}$ decrease in the kinetic energy from the solid to the liquid at $300 \mathrm{~K}$. This should be further investigated to provide detailed information on the anharmonic character of the simultaneous and competing softening and stiffening of the internal and external vibrations across the melting [27,57].

Further measurements are under planning to study the effects of density and disorder on $\left\langle E_{K}(T)\right\rangle$ in ice, to elucidate the role of both softening due to the anharmonic character of increased/decreased $\mathrm{H}$-bonding in the amorphous ices with respect to the polycrystalline phases.

CA and RS to acknowledge J. Mayers and J. Tomkinson for their seminal contributions to the electron Volt instrumental capabilities at the ISIS neutron source. G. Gorini, M. Tardocchi, E. Perelli Cippo, G. Reiter, N. J. Rhodes, E. M. Schooneveld, for joint collaboration over the years in the exploitation of methods, devices and techniques for electron Volt neutron spectroscopy. RS ackowledges R. Moreh for useful discussions. This work was supported within the CNR-STFC Agreement No. 06/20018 concerning collaboration in scientific research at the spallation neutron source ISIS.

\section{References}

[1] M.A. Adams, J. Mayers, O. Kirichek, R.B.E. Down, Phys. Rev. Lett. 98 (2007) 085301.

[2] C.J. Burnham, T. Hayashi, R.L. Napoleon, T. Keyes, S. Mukamel, G.F. Reiter, The proton momentum distribution in strongly $\mathrm{H}$-bonded phases of water: a critical test of electrostatic models, J. Chem. Phys. (2011) 135.

[3] M. Ceriotti, D.E. Manolopoulos, Phys. Rev. Lett. 109 (2012) 100604.

[4] C. Pantalei, A. Pietropaolo, R. Senesi, S. Imberti, C. Andreani, J. Mayers, C. Burnham, G. Reiter, Phys. Rev. Lett. 100 (2008) 177801.

[5] C. Andreani, D. Colognesi, J. Mayers, G.F. Reiter, R. Senesi, Adv. Phys. 54 (2005) 377.

[6] G.I. Watson, J. Phys. Condens. Matter 8 (1996) 5955

[7] J.M.F. Gunn, C. Andreani, J. Mayers, J. Phys. C Solid State Phys. 19 (1986) L835.

[8] J. MAyers, G. Reiter, Meas. Sci. Technol. 23 (2012) 045902.

[9] J. Mayers, A.C. Evans, Nuovo Cimento D Ser. 16 (1994) 737.

[10] A. Pietropaolo, R. Senesi, Phys. Rep. 508 (2011) 45.

[11] M.A. Fradkin, S.-X. Zeng, R.O. Simmons, Phys. Rev. B 49 (1994) 3197.

[12] D. Colognesi, Phys. B: Condens. Matter 406 (2011) 2723.
[13] M. Celli, D. Colognesi, M. Zoppi, Eur. Phys. J. B - Condens. Matter Complex Syst. $14(2000) 239$.

[14] C. Andreani, D. Colognesi, A. Filabozzi, M. Nardone, R. Azuah, Europhys. Lett. 37 (1997) 329.

[15] J. Dawidowski, F.J. Bermejo, M.L. Ristig, C. Cabrillo, S.M. Bennington, Phys. Rev. B 73 (2006) 144203.

[16] D. Colognesi, C. Andreani, R. Senesi, Europhys. Lett. 50 (2000) 202.

[17] C. Andreani, A. Filabozzi, M. Nardone, F.P. Ricci, J. Mayers, Phys. Rev. B 50 (1994) 12744.

[18] F. Albergamo, M. Nardone, A. Filabozzi, Phys. Rev. B 56 (1997) 14614.

[19] D.M. Ceperley, R.O. Simmons, R.C. Blasdell, Phys. Rev. Lett. 77 (1996) 115.

[20] J. Mayers, C. Andreani, D. Colognesi, J. Phys. Condens. Matter 9 (1997) 10639.

[21] D.N. Timms, R.O. Simmons, J. Mayers, Phys. Rev. B 67 (2003) 172301.

[22] A.C. Evans, J. Mayers, D.N. Timms, J. Phys. Condens. Matter 6 (1994) 4197.

[23] C. Filippi, D.M. Ceperley, Phys. Rev. B 57 (1998) 252.

[24] R. Moreh, D. Nemirovsky, J. Chem. Phys. 133 (2010) 084506.

[25] C. Andreani, E. Degiorgi, R. Senesi, F. Cilloco, D. Colognesi, J. Mayers, M. Nardone, E. Pace, J. Chem. Phys. 114 (2001) 387.

[26] T.E. Markland, B. Berne, Proc. Nat. Acad. Sci. 109 (2012) 7988.

[27] S. Habershon, T.E. Markland, D.E. Manolopoulos, J. Chem. Phys. 131 (2009) 024501.

[28] M. Ceriotti, D.E. Manolopoulos, Phys. Rev. Lett. 109 (2012) 100604.

[29] C. Andreani, D. Colognesi, E. Degiorgi, M.A. Ricci, J. Chem. Phys. 115 (2001) 11243.

[30] C. Andreani, D. Colognesi, A. Pietropaolo, R. Senesi, Chem. Phys. Lett. 518 (2011) 1.

[31] G.S. Samosvat, Y.S. Sayasov, V.T. Chuburkov, Soviet J. Exp. Theor. Phys. 27 (1968) 15

[32] L. Lin, J.A. Morrone, R. Car, M. Parrinello, Phys. Rev. Lett. 105 (2010) 110602.

[33] A. Giuliani, M.A. Ricci, F. Bruni, J. Mayers, Phys. Rev. B 86 (2012) 104308.

[34] A. Pietropaolo, C. Andreani, A. Filabozzi, R. Senesi, G. Gorini, E. Perelli-Cippo, M. Tardocchi, N.J. Rhodes, E.M. Schooneveld, J. Instrum. 1 (2006) P04001.

[35] A. Pietropaolo, R. Senesi, C. Andreani, A. Botti, M.A. Ricci, F. Bruni, Phys. Rev. Lett. 100 (2008) 127802.

[36] B. Pamuk, J.M. Soler, R. Ramirez, C.P. Herrero, P.W. Stephens, P.B. Allen, M.-V Fernandez-Serra, Phys. Rev. Lett. 108 (2012) 193003

[37] G. Reiter, C. Burnham, D. Homouz, P.M. Platzman, J. Mayers, T. Abdul-Redah, A.P. Moravsky, J.C. Li, C.-K. Loong, A.I. Kolesnikov, Phys. Rev. Lett. 97 (2006) 247801.

[38] G.F. Reiter, J.C. Li, J. Mayers, T. Abdul-Redah, P. Platzman, Braz. J. Phys. 34 (2004) 142

[39] Y. Finkelstein, R. Moreh, J. Chem. Phys. 139 (2013) 044716

[40] Y. Marechal, The Hydrogen Bond and the Water Molecule: The Physics and Chemistry of Water Aqueous and Bio-Media, Elsevier Science, 2006.

[41] C.P. Herrero, R. Ramirez, J. Chem. Phys. 134 (2011) 094510.

[42] C.P. Herrero, R. Ramirez, Phys. Rev. B 84 (2011) 224112.

[43] R. Ramirez, C.P. Herrero, Phys. Rev. B 84 (2011) 064130.

[44] C.P. Herrero, R. Ramirez, J. Chem. Phys. 137 (2012) 104505.

[45] D. Flammini, A. Pietropaolo, R. Senesi, C. Andreani, F. McBride, A. Hodgson, M.A. Adams, L. Lin, R. Car, J. Chem. Phys. 136 (2012) 024504.

[46] E.M. Schooneveld, J. Mayers, N.J. Rhodes, A. Pietropaolo, C. Andreani, R. Senesi, G. Gorini, E. Perelli-Cippo, M. Tardocchi, Rev. Sci. Instrum. 77 (2006) 5103.

[47] J. Mayers, Meas. Sci. Technol. 22 (2011) 015903.

[48] R. Senesi, A. Pietropaolo, C. Andreani, Nucl. Instrum. Methods Phys. Res. A 594 (2008) 244

[49] S. Huotari, J.A. Soininen, T. Pylkkänen, K. Hämäläinen, A. Issolah, A. Titov, J McMinis, J. Kim, K. Esler, D.M. Ceperley, M. Holzmann, V. Olevano, Phys. Rev. Lett. 105 (2010) 086403.

[50] L. Lin, J.A. Morrone, R. Car, M. Parrinello, Phys. Rev. B 83 (2011) 220302.

[51] A. Pietropaolo, R. Senesi, C. Andreani, J. Mayers, Braz. J. Phys. 39 (2009) 318.

[52] G.F. Reiter, R. Senesi, J. Mayers, Phys. Rev. Lett. 105 (2010) 148101.

[53] R. Senesi, D. Flammini, A.I. Kolesnikov, Éamonn D. Murray, G. Galli, C. Andreani, J. Chem. Phys. 139 (2013) 0745043251.

[54] C. McBride, J.L. Aragones, E.G. Noya, C. Vega, Phys. Chem. Chem. Phys. 14 (2012) 15199.

[55] G. Romanelli, M. Ceriotti, D.E. Manolopoulos, C. Pantalei, R. Senesi, C. Andreani J. Phys. Chem. Lett. 4 (19) (2013) 3251, http://dx.doi.org/10.1021/jz401538r.

[56] D. Colognesi, Phys. B Condens. Matter 406 (2011) 2723.

[57] X.-Z. Li, B. Walker, A. Michaelides, Proc. Nat. Acad. Sci. (2011). 